\title{
Research on Optimization of Vehicle ABS System Based on Fuzzy PID Control
}

\author{
TANG Yan ${ }^{1, a}$, ZHAO Yu-peng ${ }^{2, b}$ \\ ${ }^{1,2}$ GuiLin University of Electronic Technology,Guangxi province,China \\ a1195957256@qq.com,'958242252@qq.com
}

Keywords: ABS, Fuzzy PID control, Intelligent Algorithm, Multi-objective optimization.

\begin{abstract}
ABS quality directly affects the stability of vehicle braking and traffic safety, the current ABS in the robustness and adaptability need to be improved. Aiming at the shortcomings of the traditional fuzzy control that due to the lack of integral part, so the system lack of steady state precision, the single model ABS system model is established in MATLAB / Simulink, and the adaptive fuzzy PID controller is designed by the improved algorithm. Input different road spectrum, in different speed conditions, then analysis of ABS control parameters of real-time multi-objective optimization of the braking effect. The results show that the responses speed of the system is $60 \%$ higher than that the traditional fuzzy control, and the steady - state precision and robustness are also improved obviously.

\section{Introduction}

With the popularity of automobiles and the rapid development of vehicle electronics technology, to enhance the active safety of modern cars has become one of the hot issues in the industry. Excellent ABS function is the protection of vehicle to play the best ground effect. ABS has become the standard equipment for all types of cars .As the time of emergency braking is extremely short, it is seriously affected by many factors, and the related dynamic control is a complex nonlinear problem. At present, most of the vehicle ABS system controller lacks the adaptive characteristic, especially in the automobile emergency braking process is difficult to obtain the ideal corresponding speed and the robustness is poor. The statistical analysis shows that the domestic ABS controller design is divided into two categories: one is the conventional PID control, there are defects that the braking parameters do not change with the control object. The second is the conventional fuzzy control, the lack of integral links, and once the fuzzy rules are determined, The control parameters can not be adjusted online. In this paper, the fuzzy PID controller with ideal adaptive characteristic is designed by the improved algorithm. The simulation results show that the braking control effect is improved obviously compared with the traditional control method. The research results are helpful to promote the progress of intellectual property rights and provide the theoretical basis for the application of intelligent control algorithm in ABS system.
\end{abstract}

\section{Target vehicle ABS system model}

Single Wheel Vehicle Dynamics Model. Simplified single-wheel dynamics model shown in Figure $1^{[1]}$ : 


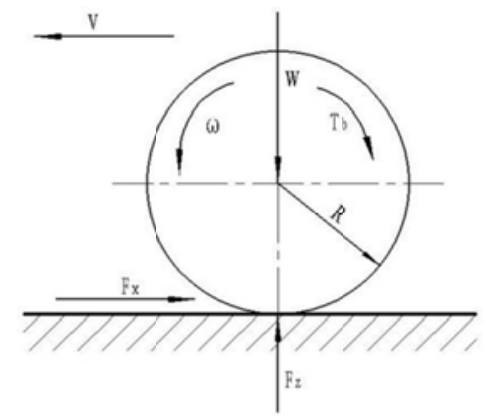

Fig.1 Force analysis on wheel braking

$F_{\mathrm{x}}$-ground braking force; $T_{\mathrm{b}}$-brake torque; $R$-wheel radius; $V$-wheel center speed; $W$-vertical load; $F_{\mathrm{z}}$-ground-to-wheel normal reaction force.

Ignoring the influence of air resistance and wheel rolling resistance, the system equation of motion is as follows:

Vehicle motion equation:

$$
m \frac{d v}{d t}=-F_{X} .
$$

Wheel motion equation:

$$
I_{\omega} \frac{d \omega}{d t}=F_{x} R-T_{b} .
$$

Ground Brake:

$$
F_{x}=\mu F_{z} .
$$

$m$-1/4 vehicle quality; $\omega$-wheel angular velocity; $I_{\omega}$ - wheel moment of inertia; $\mu$ - longitudinal adhesion coefficient.

Slip rate calculation. In the vehicle braking process, the degree of sliding isi usually expressed by the slip rate, which is defined as:

$$
s=\frac{v-\omega \cdot R}{v} \times 100 \%=\left(1-\frac{\omega \cdot R}{v}\right) \times 100 \% .
$$

$v$ is the instantaneous speed of the vehicle; $\omega$ is the rotational speed of the wheel; $R$ is the wheel rolling radius.

Tire model. A bilinear tire model describing the nonlinear relationship between the pavement adhesion coefficient and the wheel slip rate is used. The expression is as follows:

$$
\mu= \begin{cases}\frac{\mu_{h}}{s_{0}} \cdot s_{b} & s_{b} \leq s_{0} \\ \frac{\mu_{h}-\mu_{g} \cdot s_{0}}{1-s_{0}}-\frac{\mu_{h}-\mu_{g}}{1-s_{0}} \cdot s_{b} & s_{b}>s_{0}\end{cases}
$$

$\mu_{h}$-peak adhesion coefficient; $\mu_{\mathrm{g}}$-wheel lock corresponding to the adhesion coefficient; $s_{0}$ -peak corresponding slip rate.

Using the bilinear tire model to simulate three typical road conditions: dry road, wet road and ice and snow pavement, the parameters in Table 1. 
Tab.1 Parameters of various road surfaces

\begin{tabular}{|c|c|c|c|}
\hline Pavement type & $s_{0}$ & $\mu_{h}$ & $\mu_{g}$ \\
\hline Dry road & 0.2 & 0.8 & 0.6 \\
\hline Wet road & 0.13 & 0.78 & 0.55 \\
\hline Ice and snow & 0.05 & 0.2 & 0.15 \\
\hline
\end{tabular}

Brake system model. ABS system is mainly composed of hydraulic transmission system and brake parts. For the real-time simulation of the brake pressure and the electromagnetic valve current changes between the law, according to the control principle of the brake force regulator, the hydraulic drive system is simplified as a solenoid valve and an integral part ${ }^{[2]}$ ignoring the solenoid valve spring nonlinear factors and pressure transmission delay, the transfer function Can be expressed as:

$$
G(s)=\frac{K}{s \cdot(T s+1)}
$$

$K$ is the gain of the system, $K=100 ; T$ is the system time constant, $T=0.01$.

The brake model describes the mechanical relationship between the braking torque and the brake fluid pressure. In the modeling, in order to facilitate the study of the control algorithm, it can be assumed that the brake is the ideal component, ignoring the hysteresis and the temperature change caused by the impact of the braking torque can be seen as a linear function of the brake pressure, the brake equation Expressed as:

$$
T_{b}=K_{P} \cdot p
$$

$T_{b}$ - brake torque; $p$ - brake pressure; $K_{p}$ - Brake energy efficiency factor.

Simulation system block diagram. Vehicle ABS control system to slip rate as the control target, by adjusting the slip rate in real time, so that the slip rate is always within the ideal slip range to ensure that the vehicle to obtain the best braking performance and direction of the control Stability [3]. Taking the adaptive fuzzy PID control system as an example, the system compares the difference between the actual slip rate and the ideal slip rate and the change rate of the difference as the input of the adaptive fuzzy PID controller. The controller output is the braking force adjustment value, Adjust the value and then through the braking system to pass the braking force to the wheel, thus controlling the wheel slip rate, so that it is controlled in the ideal slip range. The system block diagram shown in Figure 2

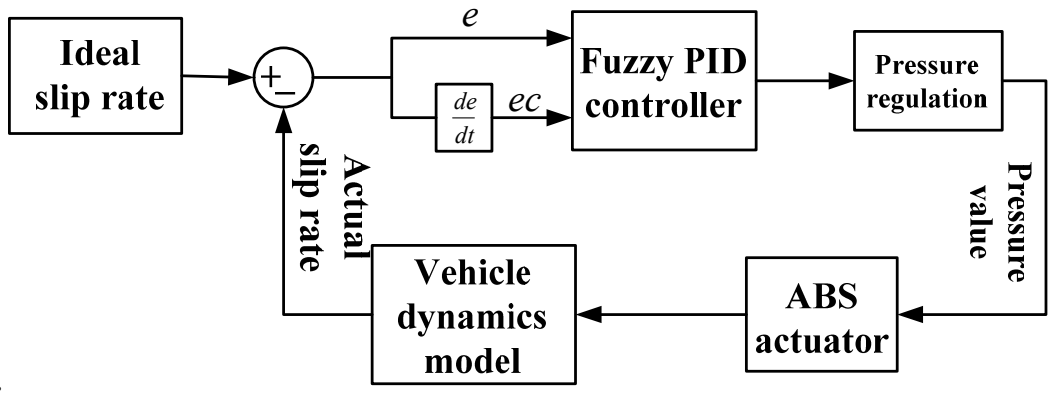

Fig.2 ABS fuzzy PID system block diagram 


\section{Design of Parameter Adaptive Fuzzy PID Controller}

Parameter Adaptive Fuzzy PID Control System Structure. The adaptive fuzzy PID controller uses the error (e)and the error rate of change(ec) as input. According to the fuzzy control principle, the three parameters of $k_{p}, k_{i}$ and $k_{d}$ are adjusted on-line to meet the changes of the control parameters with different e and ec changes Requirements, so that the accused object has a good dynamic performance ${ }^{[4]}$.

The adaptive fuzzy PID control system is mainly composed of two parts: PID controller and fuzzy reasoning parameter adjustment system ${ }^{[5]}$. Its structure shown in Figure 3.

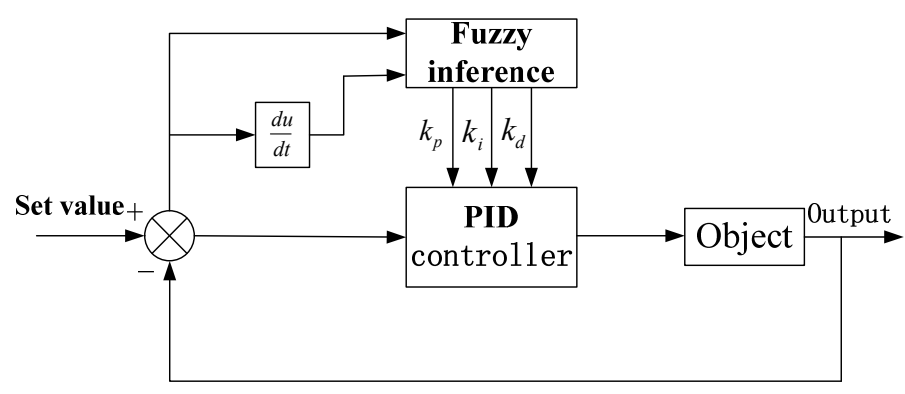

Fig.3 Adaptive fuzzy PID control system structure

PID parameter adjustment principle. Parameter adaptive adjustment Fuzzy PID control is based on the conventional PID control, through the online adjustment parameters $\Delta k_{p}, \Delta k_{i}$ and $\Delta k_{d}$, to achieve the system of adaptive fuzzy PID control, the adjustment formula is as follows:

$$
\begin{aligned}
& k_{p}=k_{p}{ }^{\prime}+\Delta k_{p} . \\
& k_{i}=k_{i}{ }^{\prime}+\Delta k_{i} . \\
& k_{d}=k_{d}{ }^{\prime}+\Delta k_{d} .
\end{aligned}
$$

$k_{p}{ }^{\prime}, k_{i}{ }^{\prime}$ and $k_{d}{ }^{\prime}$ are the initial setting of the PID control parameters.

Determine the variable domain and membership function. First, according to the principle of fuzzy PID control parameter adjustment, the fuzzy controller structure is determined as two input and three outputs, that is, the input variable is the error (e)and the error rate of change (ec), and the output is the three parameters $\Delta k_{p}, \Delta k_{i}$ and $\Delta k_{d}$ of the PID controller. The fuzzy reasoning type is based on Mamdani type fuzzy reasoning system. The fuzzy domain of e and ec is defined as [-6,6], and the fuzzy quantization factor takes $k_{e}=12, k_{e c}=1.2$. The fuzzy state is divided into seven grades: Positive Big(PB), Positive Medium(PM),Positive Small(PS),zero(ZE), Negative Small(NS), Negative Medium(NM), Negative Big(NB), fuzzy set $\{\mathrm{NB}, \mathrm{NM}, \mathrm{NS}, \mathrm{ZE}, \mathrm{PS}, \mathrm{PM}, \mathrm{PB}\}$. The fuzzy domain of the output variables $\Delta k_{p}, \Delta k_{i}$ and $\Delta k_{d}$ is defined as [0,1]. The fuzzy state is divided into five levels: Zero(ZE), Positive Zero(PO), Positive Small(PS),Positive Medium(PM), Positive Big (PB), fuzzy set $\{Z E, P O, P S, P M, P B\}$.Language variables are selected with a highly sensitive trimf membership function type.

Establish control rules and determine reasoning. According to $k_{p}, k_{i}$ and $k_{d}$ three parameters on the impact of the output characteristics to develop fuzzy rules, when the beginning of the brake, e has a great value, should choose a larger $k_{p}$ and smaller $k_{d}$, to avoid the system overshoot is too large, and ensure that the system has a good tracking performance. At the same time we should reduce the integral effect, usually take $k_{i}=0$; When $e$ and $e c$ has a medium value, in order to reduce the system overshoot, should take a smaller $k_{i}, k_{p}$ and $k_{d}$ take moderate value to ensure the system response speed ; And when $e$ has a smaller value, in order to make the system has good 
steady-state performance, should increase $k_{p}$ and $k_{i}, k_{d}$ take moderate. The specific fuzzy control rules are shown in Table 2.

The establishment of fuzzy rules using "if-then" relationship, the fuzzy rules as an example:

Rule1: if e is NB and ec is NB thanis PM.

Rule2: if e is NB and ec is NM thanis PB.

Simulation and analysis. In the three typical road conditions of dry road, wet road, ice and snow, the simulation system is simulated by conventional fuzzy control and adaptive fuzzy PID control. The simulation is set to $20 \mathrm{~s}$. Get the slip rate curve and wheel speed, body speed curve, as shown in Figure $8 \sim 10$. Pavement simulation parameters as shown in Table 1, braking time and braking distance comparison Table 2, vehicle simulation parameters as shown in Table 3.

Tab.2 Braking time and braking distance

Tab.3vehicle simulation parameters

\begin{tabular}{ccccc}
\hline \multirow{2}{*}{$\begin{array}{c}\text { Pavement } \\
\text { type }\end{array}$} & \multicolumn{2}{c}{ Fuzzy controller } & \multicolumn{2}{c}{ Fuzzy PID controller } \\
\cline { 2 - 5 } & Braking time & distance & Braking time & distance \\
\hline Dry road & 3.38 & 41.3 & 3.23 & 39.2 \\
Wet road & 3.54 & 47.1 & 3.32 & 40.6 \\
Ice and snow & 15.57 & 169.5 & 15.69 & 170.3 \\
\hline
\end{tabular}

\begin{tabular}{ccc}
\hline$V_{0}$ & $m$ & $R$ \\
\hline $25 \mathrm{~m} / \mathrm{s}$ & $395 \mathrm{~kg}$ & $0.31 \mathrm{~m}$ \\
\hline$I_{\mathrm{w}}$ & $\mathrm{g}$ & \\
\hline $2.1 \mathrm{~kg} \cdot \mathrm{m}^{2}$ & $9.8 \mathrm{~m} / \mathrm{s}^{2}$ & \\
\hline
\end{tabular}

In Fig. 4 6, (a) shows the comparison between the conventional fuzzy control and the improved fuzzy PID control slip rate curve in each case. (B) shows the vehicle speedl and the wheel speed curve in the conventional fuzzy control of each condition, (c) Increased speed and wheel speed curve under improved fuzzy PID control in each condition.

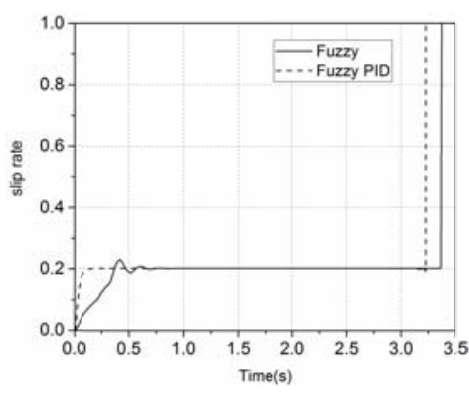

(a)

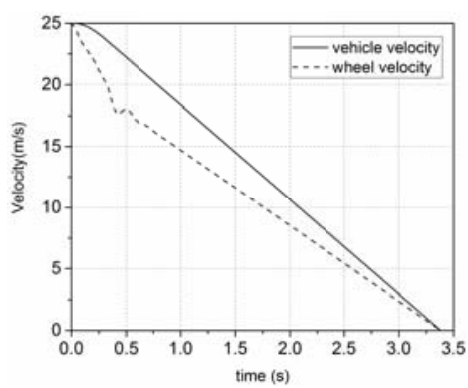

(b)

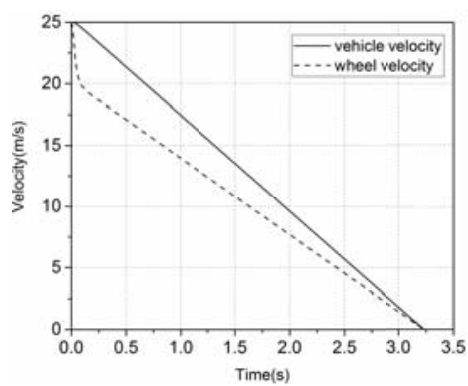

(c)

Fig.4 Comparison of simulation results of linear braking process on dry road

On the dry road, the conventional fuzzy control and the fuzzy PID control can make the slip rate curve reach the steady state at the ideal slip value. However, the fuzzy PID control is $60 \%$ higher than the conventional fuzzy control in the fastness of the system response. , In the braking time, fuzzy PID than the conventional fuzzy control increased by $4.43 \%$.

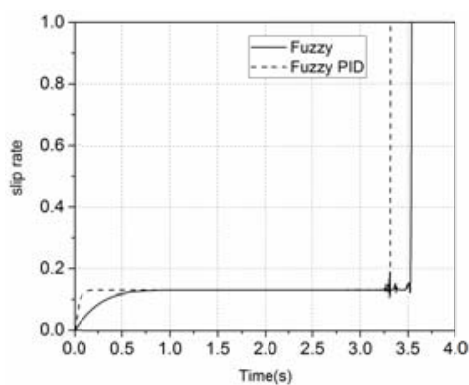

(a)

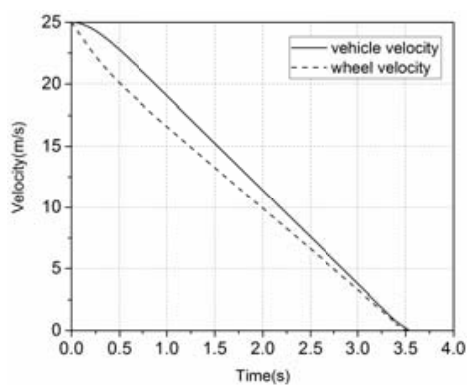

(b)

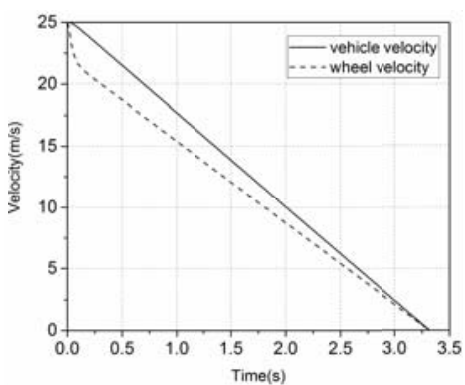

(c)

Fig.5 Comparison of simulation results of linear braking process on wet road

On the wet road, the same slip rate curve is stable at both the ideal slip value of 0.13 , and the 
wheel speed are not volatile. The conventional fuzzy control on the braking time is $3.54 \mathrm{~s}$, but the improved fuzzy PID control is $3.32 \mathrm{~s}$, which increases $6.2 \%$ in braking performance.

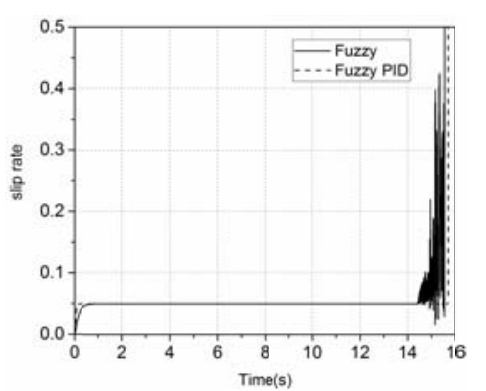

(a)

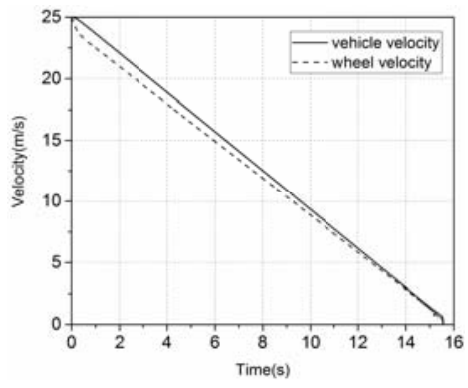

(b)

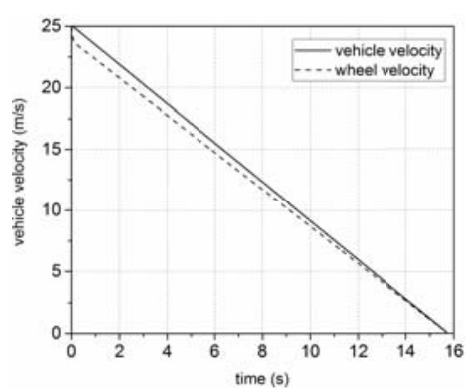

(c)

Fig.6 Comparison of simulation results of linear braking process on ice and snow road

On the snow and ice road, the slip curve of conventional fuzzy control fluctuates at the time of stopping, and the steady state accuracy is poor, which is due to the lack of integral part. But the improved fuzzy PID controller solves this problem.

\section{Summary}

In view of the complex and volatile dynamic vehicle control, the modern control theory based on the model design has been difficult to adapt to its control requirements, and the intelligent control algorithm has become the application trend. The improved fuzzy PID controller has the ideal real-time optimization of the control parameters of the vehicle ABS system. The experimental results show that the braking response time is increased by $60 \%$ under the same conditions; the braking robustness is also improved; the research results are ABS system control provides a theoretical basis.

\section{References}

[1]YU Fan,LIN Yi.Vehicle System Dynamics[M].Beijing:China Machine Press, 2005.7 .

[2]LI Bo-quan,DONG Xian-lun,HE Ren,etal.Simulation of vehicel ABS based on electronic mechanical brake system and road surface automatic identification[J].Machinery Design and Manufacture,2009,9(9):186 188.

[3]LU Yi,WU Jia-wei,LU Jie,etal.Semi Physical Simulation Test of Turning Braking of Bus ABS Based on Fuzzy PID[J].Chinese Hydraulic and Pneumatic,2016, (11): 55 60.

[4]WU Ling,WEN Kai,DONG Min,etal.Application of Adaptive Fuzzy PID Control on the Anti-Lock Braking System of Automobiles[J].Techniques of Automation and Applications,20 16,35(1):27 31 .

[5]Hu Qiguo,Ren Long.Simulation of Fuzzy PID Control for Vehicle Anti-Lock Braking Sy stem[J].Journal of Chongqing Jiaotong University(Natural Science), 2012, 31(2)344 348.

[6]WANG Hui, Yang Lin,Simulation of Automotive ABS Using Fuzzy Self turning PID Con -trol[J].Journal of Transportation Systems Engineering and Information Technology ,2012,12 (5): $52 \sim 57$.

[7]Liu Wu-tong,Duan Min,Sun Ming-jiang,etal.Simulation of Vehicle ABS Fuzzy PID Contr ol Based on Road Surface Automatic Identification[J].Agricultural Equipment \& Vehicle En gineering, 2016, 54(12):14 18.

[8]Wang Shu-yan,Shi Yu,Feng Zhong-xu.A Method for Controlling Loading System Based on a Fuzzy PID Controller[J].Mechanical Science and Technology for Aerospace Engineerin g, 2011, 1(1): 166 172. 